\title{
Un modelo matemático para la reducción del tiempo de compostaje
}

\section{(A mathematical model for reducing the composting time)}

\author{
Estefanía Larreategui ${ }^{1}$, Carlos Banchón ${ }^{2}$
}

\begin{abstract}
Resumen:
El medio ambiente es todavía afectado por el uso inadecuado de residuos orgánicos aunque una cultura de reciclaje y aprovechamiento ha sido promovida en Ecuador para disminuir la huella de carbono. El compostaje, técnica de digestión de materia orgánica, la cual tradicionalmente demora entre 16 y 24 semanas, es todavía ineficiente de aprovechar. Es por esto que, el presente trabajo se refiere a la optimización del proceso de compostaje tanto en calidad como en el tiempo de producción. Las variables estudiadas fueron: tipo de residuo (frutas y vegetales) y tipo de bioacelerador (levaduras y microorganismos autóctonos). Mediante el Diseño Factorial Completamente Aleatorio $2^{2}$ (DFCA) se obtuvo un compost de calidad en 7 semanas de procesamiento. Los factores de control como temperatura, densidad, humedad, $\mathrm{pH}$, relación carbono-nitrógeno permitieron comprobar las mejores condiciones para el compostaje en la comunidad San Gabriel del Baba (Santo Domingo de los Colorados, Ecuador). Como resultado de este estudio se obtuvo un modelo matemático de superficie que explica la relación entre la temperatura y el tiempo de digestión de la materia orgánica.
\end{abstract}

Palabras clave: compostaje, digestión, optimización, residuos, materia orgánica.

\begin{abstract}
:
The environment is still affected by the inappropriate use of organic matter waste, but a culture of recycling and reuse has been promoted in Ecuador to reduce carbon footprint. The composting, a technique to digest organic matter, which traditionally takes $16-24$ weeks, is still inefficient to use. Therefore, this paper concerns the optimization of the composting process in both quality and production time. The variables studied were: type of waste (fruits and vegetables) and type of bioaccelerator (yeast and indigenous microorganisms). By using a full factorial random design $2^{2}$, a quality compost was obtained in 7 weeks of processing. Quality factors as temperature, density, moisture content, $\mathrm{pH}$ and carbon-nitrogen ratio allowed the best conditions for composting in the San Gabriel del Baba community (Santo Domingo de los Colorados, Ecuador). As a result of this study, a mathematical surface model which explains the relationship between the temperature and the digestion time of organic matter was obtained.
\end{abstract}

Keywords: composting, digestion, optimization, residues, microorganisms

\section{Introducción}

A nivel mundial, se considera al manejo de residuos sólidos un problema ambiental que afecta a la población urbana y rural. La falta de infraestructura, presupuesto, técnicos o programas de aprovechamiento de residuos es todavía latente en algunos países. En Ecuador se generan cerca de 7,423 toneladas de residuos sólidos al día, del cual el $71.4 \%$ corresponde a materia orgánica

\footnotetext{
${ }_{1}^{1}$ Universidad de Las Américas, Ingeniería Ambiental, Quito - Ecuador (rlarreategui@udlanet.ec)

2 Universidad de Las Américas, Ingeniería Ambiental, Quito - Ecuador (cbanchon@udla.edu.ec)
} 
que pudiera ser utilizada en procesos de compostaje (Organización Panamericana de la Salud, 2002). En Ecuador, se estima que existe un reciclaje total de los residuos (formal e informal) del $14 \%$ y el costo promedio del manejo de los residuos sólidos por tonelada es de USD 43.05 (Soliz, 2011).

El compost mejora propiedades del suelo como su estructura, capacidad hídrica y de drenaje, además de incrementar su contenido de materia orgánica y poblaciones de microorganismos (Antil \& Raj, 2012; Insam, Franke-Whittle, \& Goberna, 2009; Termorshuizen, Moolenaar, Veeken, \& Blok, 2004; Xu et al., 2012). Es conocido también que el compost incrementa la calidad y productividad de plantas porque incrementa la resistencia contra patógenos (Insam, FrankeWhittle, \& Goberna, 2009; Termorshuizen, Moolenaar, Veeken, \& Blok, 2004; Xu et al., 2012). Por tanto, el objetivo del presente trabajo fue implementar un sistema de compostaje utilizando residuos orgánicos de una población ubicada en una zona arbórea húmeda de Ecuador. Para este propósito, se planteó el diseño de una unidad experimental de compostaje por pilas, la identificación de qué tipos de residuos orgánicos y bioaceleradores de digestión que son necesarios para la obtención de compost de calidad, y la aplicación de un diseño experimental estadístico para determinar las condiciones óptimas de compostaje.

\section{Metodología}

\subsection{Compostaje por pilas}

El lecho de compostaje fue construído a base de madera ( $0.5 \mathrm{~m}$ de ancho, $1.0 \mathrm{~m}$ de largo y $1.0 \mathrm{~m}$ de alto). En cada lecho se colocaron: $5 \mathrm{~cm}$ de virutas de madera en el fondo de acuerdo a Bougnom et al., 2010, $10 \mathrm{~cm}$ de materia orgánica seca y $15 \mathrm{~cm}$ de materia orgánica húmeda (frutas o vegetales) y $5 \mathrm{~cm}$ de suelo del bosque como material de cobertura. Esta unidad fue ubicada en San Gabriel del Baba (Santo Domingo de los Colorados, Ecuador) a una altitud promedio de 600 metros sobre el nivel del mar, temperatura entre $18-22^{\circ} \mathrm{C}$, precipitación entre 2000-4000 mm por año; el lugar de experimentación es considerado como bosque muy húmedo premontano (b.m.h.P.M.) según Restrepo, N. (2007).

\subsection{Aislamiento de microorganismos eficientes autóctonos}

Se aislaron microorganismos del suelo del bosque sub-tropical húmedo de San Gabriel del Baba mediante la adaptación del método aplicado por Chantal et al. (2013). Para preparar una solución activada de microorganismos (Effective Microorganism Activated Solution, EMAS), se preparó un medio de cultivo a base de arroz cocido sin sal, extractos de carne y de pescado. El medio estéril se colocó dentro de envases plásticos para posteriormente enterrarlos en un talud húmedo. Al cabo de dos semanas, se retiraron los envases del talud y se preparó una solución madre con los EMAS aislados. Para esto, se mezclaron los EMAS en un envase de $5 \mathrm{~L}$ con una porción de arroz 
cocido, $0.75 \mathrm{~L}$ de melaza y $2 \mathrm{~L}$ de agua pura hasta homogeneización. La solución fue conservada a $4^{\circ} \mathrm{C}$.

\subsection{Medición de parámetros físico-químicos del compost}

La temperatura del compost fue tomada en la mitad de cada lecho de compostaje con termómetro de mercurio. Se disolvió $10 \mathrm{~g}$ de muestra en $25 \mathrm{~mL}$ de agua destilada y con agitación constante se midió el pH con un dispositivo portátil (FieldScout). La humedad fue determinada mediante el método gravimétrico ASTM D4959. Para determinar la densidad aparente se utilizó una probeta plástica para la toma de muestra en lugar de un cilindro metálico como lo indica el método ASTM D 2937-00. El análisis químico determinó lo siguiente: carbono, nitrógeno y materia orgánica. Estos parámetros fueron medidos mediante espectrometría de masas y Kjeldhal (Agrobiolab No. 46464).

\subsection{Diseño experimental}

Se aplicó el Diseño Factorial Completamente Aleatorio (DFCA) $2^{2}$ el cual fue elaborado y analizado mediante el programa estadístico JMP (versión 9.0) y el paquete R-project (versión 3.0.1, paquete rsm). En la Tabla 1, dos variables categóricas $\left(\mathrm{X}_{1} \mathrm{y}_{2}\right)$ fueron seleccionadas: tipos de residuos de pronta digestión, frutas y vegetales; y dos tipos de bioaceleradores del compostaje: una mezcla de levaduras comerciales y bebida gaseosa altamente azucarada $(L+G), y$ microorganismos eficientes autóctonos (EMAS). En la Tabla 2, se combinaron aleatoriamente las variables y sus niveles para generar un total de cuatro tratamientos (dos réplicas). En cada tratamiento (lecho de compostaje) se analizó la evolución del proceso durante 7 semanas mediante el control de temperatura, $\mathrm{pH}$, humedad, densidad aparente, relación carbono-nitrógeno, y materia orgánica.

Tabla 1. Matriz de factores y sus niveles para la experimentación.

\begin{tabular}{|c|c|c|}
\hline Factores de estudio $(X)$ & Nivel bajo $(-1)$ & Nivel bajo $(+1)$ \\
\hline Tipo de residuo, $X_{1}$ & Frutas & Vegetales \\
\hline Tipo de bioacelerador, $\mathrm{X}_{2}$ & $\mathrm{~L}+\mathrm{G}$ & EMAS \\
\hline
\end{tabular}

Tabla 2. Matriz para la experimentación en 4 tratamientos: combinación de factores y sus niveles de acuerdo al DFCA $2^{2}$.

\begin{tabular}{|c|c|c|}
\hline Tratamiento (lecho) & $\mathrm{X}_{1}$ & $\mathrm{X}_{2}$ \\
\hline $1(\mathrm{~A})$ & -1 (Frutas) & $-1(\mathrm{~L}+\mathrm{G})$ \\
\hline $2(\mathrm{~B})$ & -1 (Frutas) & $+1($ EMAS) \\
\hline $3(\mathrm{C})$ & +1 (Vegetales) & $-1(\mathrm{~L}+\mathrm{G})$ \\
\hline $4(\mathrm{D})$ & +1 (Vegetales) & $+1($ EMAS) \\
\hline
\end{tabular}




\section{Resultados}

La combinación de los tipos de residuos y de bioaceleradores en el compostaje tuvo un considerable efecto en la temperatura, densidad y humedad de los cuatro lechos A, B, C y D, así como en su composición química. En la Figura 1 se presenta la variación de temperatura en función de cuatro tratamientos y siete semanas de compostaje, en donde existieron incrementos de hasta $34{ }^{\circ} \mathrm{C}$ por encima de la temperatura inicial.

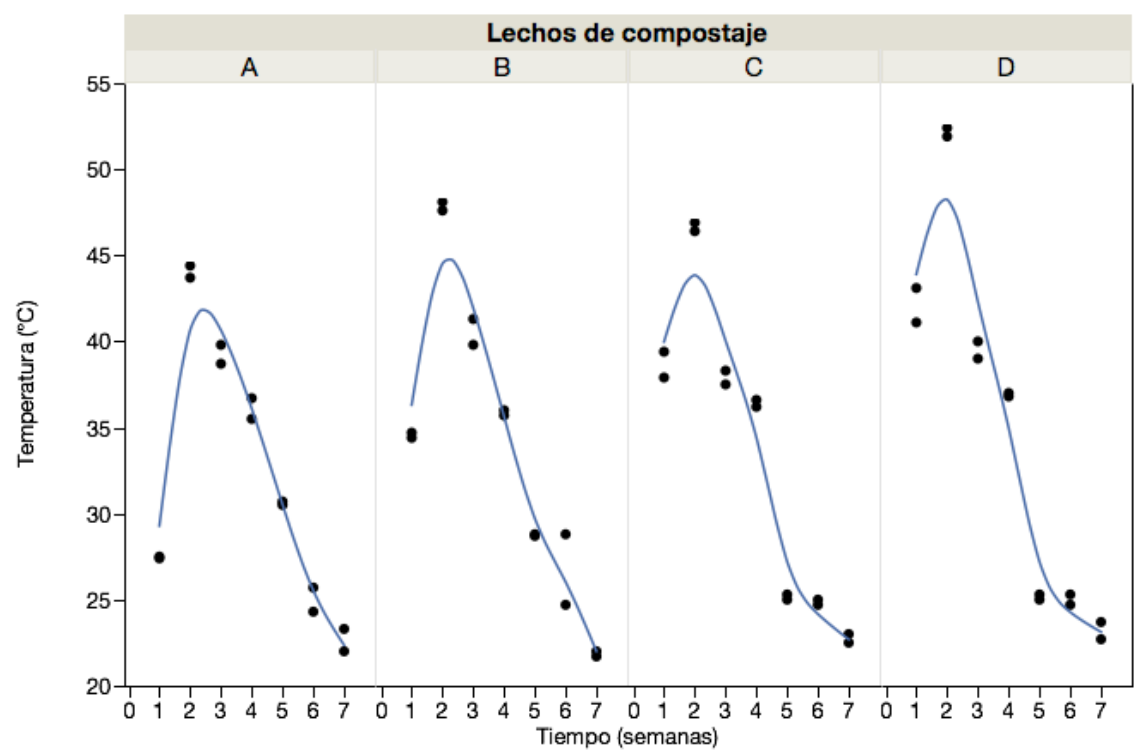

Figura 1. Variación de la temperatura en función del tiempo (7 semanas) y de los cuatro lechos de compostaje A (Combinación de frutas y levaduras), B (Combinación de frutas y EMAS), C (Combinación de vegetales y levaduras) y D (Combinación de vegetales y EMAS).

En la Figura 2 se presenta la variación del promedio de la densidad y humedad de la materia orgánica en función del tiempo para todos los cuatro tratamientos.

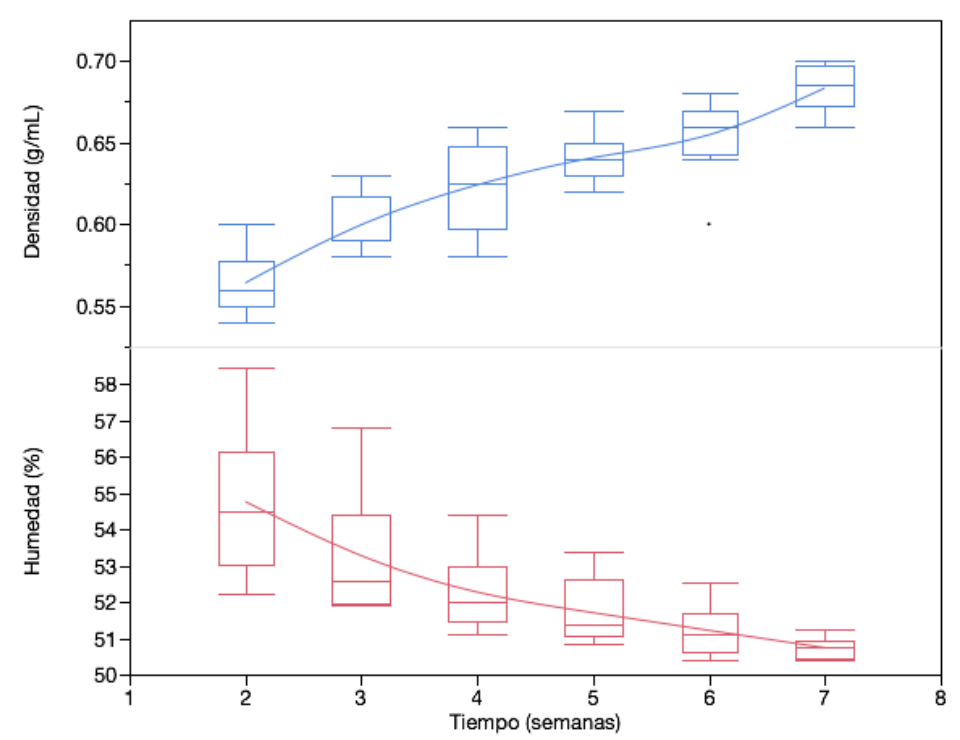

Figura 2. Promedio de variación de la densidad y humedad de los cuatro lechos ( $A, B, C$ y $D)$ en función del tiempo (7 semanas). 
El análisis químico de la materia orgánica inicial (muestra blanco) y de los cuatro tratamientos después de siete semanas de compostaje se muestra en la Figura 3.

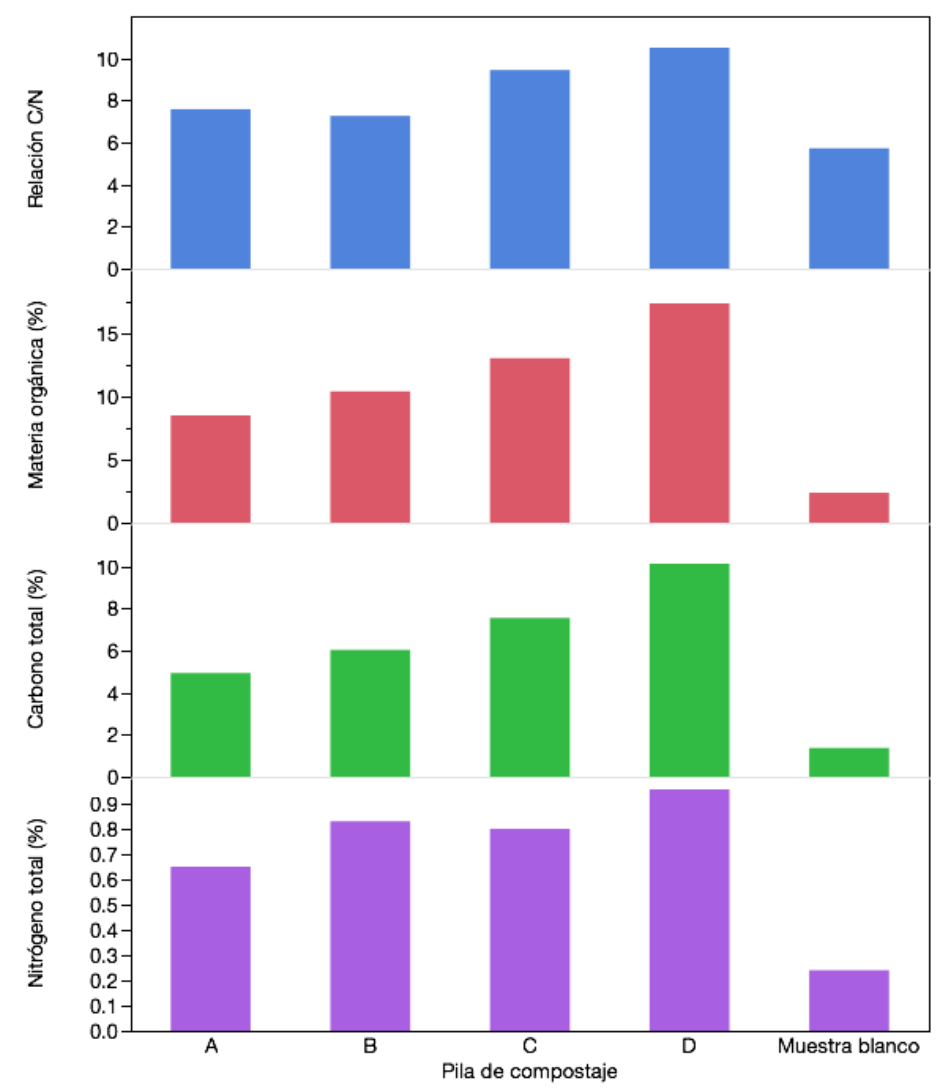

Figura 3. Análisis químico en los tratamientos A, B, C, D y la muestra blanco al cabo de 7 semanas.

De los datos de temperatura obtenidos en los cuatro tratamientos, se estableció un modelo matemático polinomial en función del tiempo (Figura 4). Este modelo fue elaborado en el software JMP (versión 9.0). El coeficiente de determinación de este modelo fue de 0.834 y el análisis de varianza para todos los términos de la Ecuación (1) tuvieron un $p<0.0001$. En la Figura $4 B$ también se reportan los residuales de las mediciones de temperatura.

$$
\text { Ec. (1) } T=66.1192-7.5168^{*} t-0.6415^{\star}(t-4)^{2}+0.5857^{*}(t-4)^{3}
$$

\section{Discusión}

La variación de temperatura de hasta $34{ }^{\circ} \mathrm{C}$, según la Figura 1, indica que los residuos orgánicos estuvieron sometidos a las fases de degradación microbiana reportadas en la literatura para la descomposición de azúcares, almidones y proteínas (Adediran, Taiwo, \& Sobulo, 2003; Antil \& Raj, 2012). De los resultados obtenidos, se puede deducir que todos los cuatro tratamientos cumplieron con las fases necesarias para un correcto desarrollo microbiano del compostaje: mesofílica y termófila (Adediran, Taiwo, \& Sobulo, 2003; Agnew \& Leonard, 2003; Heribert Insam, Franke-Whittle, \& Goberna, 2010). Todos los tratamientos se encontraron dentro de los rangos aceptables de humedad durante siete semanas, ya que la humedad (entre 50-60\%) es un factor crítico para la descomposición de la materia orgánica según la Figura 2 (Insam, Franke-Whittle, \& Goberna, 
2009). Los tratamientos $A$ y $B$ registraron los porcentajes más altos de humedad durante las primeras semanas, debido a que en estos lechos se trabajó con desechos de frutas. En relación a la composición de $\mathrm{C} / \mathrm{N}$, Figura 3, el mayor incremento se produjo en el lecho D con una relación final de 10.56. La relación $\mathrm{C} / \mathrm{N}$ óptima para un compost totalmente maduro es cercana a 10 , similar a la del humus. Generalmente, cuando la relación $\mathrm{C} / \mathrm{N}$ es menor a 20 , se considera que el compost es estable o maduro. Según la Figura 3, la relación $\mathrm{C} / \mathrm{N}$ para las cuatro muestras de compost es cercana a 10, indicando nivel de madurez del compost (Jusoh, Manaf, \& Latiff, 2013).

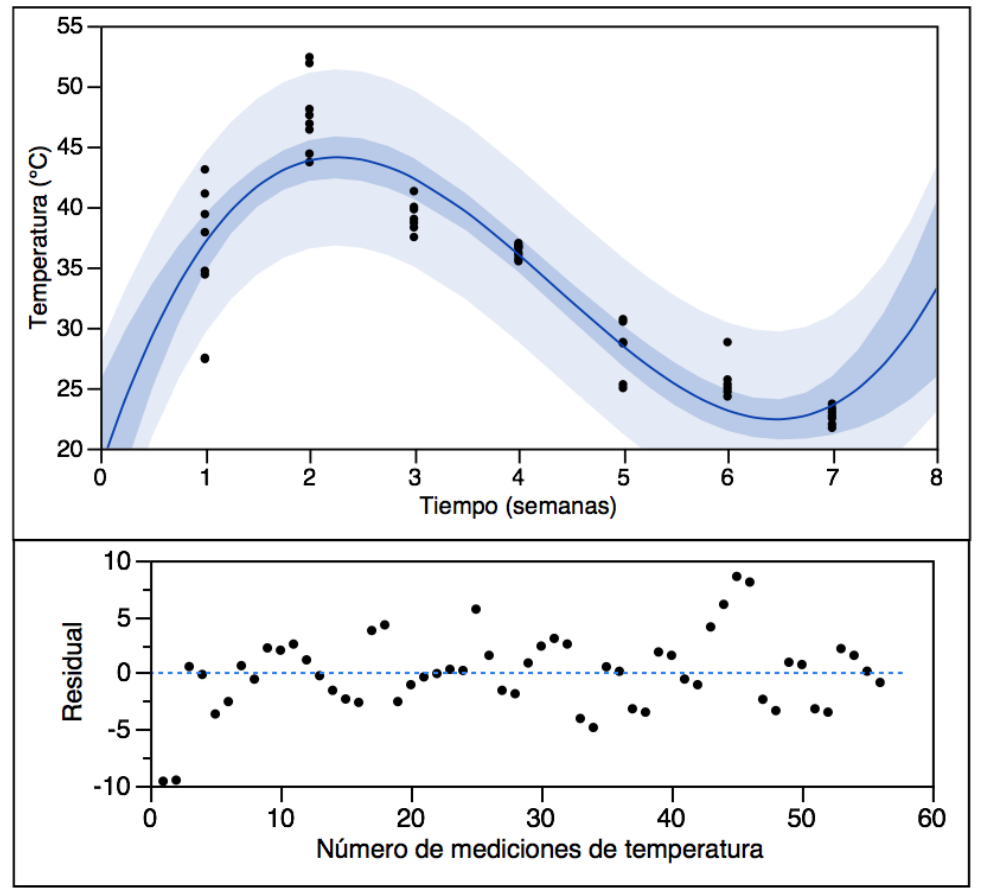

Figura 4. Ajuste polinomial: (A) modelo matemático para temperatura y tiempo de compostaje, y (B) residuales del ajuste polinomial en función de 56 mediciones de temperatura en siete semanas.

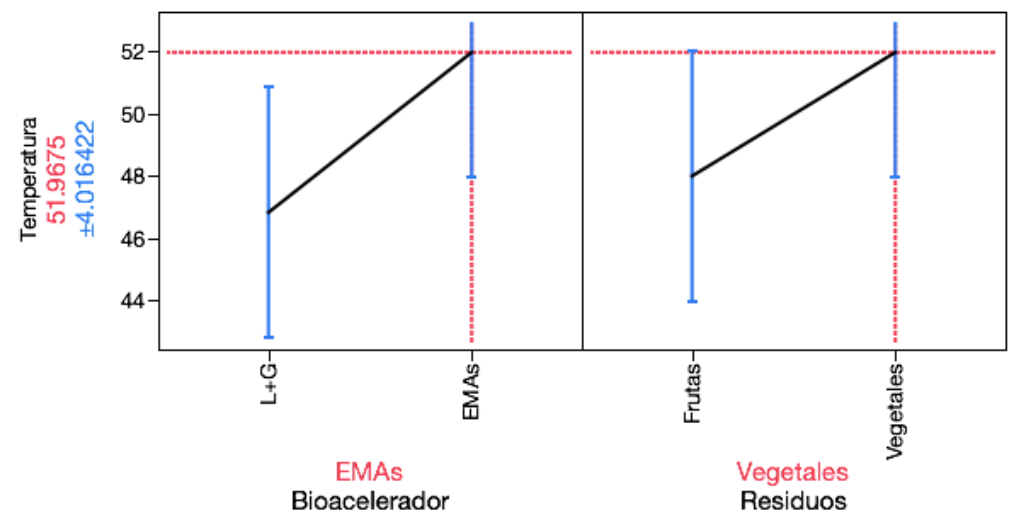

Figura 5. Efectos principales de los factores tipo de residuos y tipo de bioaceleradores.

El análisis de efectos principales de la Figura 5 indica qué nivel del factor de estudio genera mayor incidencia en el tratamiento. La temperatura del compostaje fue la respuesta tomada en cuenta para el análisis estadístico porque denota una gran relación el proceso de maduración y degradación de la materia orgánica (Adediran, Taiwo, \& Sobulo, 2003; Jusoh, Manaf, \& Latiff, 2013). De acuerdo con la Figura 5, según el tipo de residuo, cuando se adicionan desechos de 
vegetales la temperatura alcanza promedios más altos que cuando se trabaja con desechos de frutas. Para el tipo de bioacelerador, cuando se adicionan EMAS el efecto en la temperatura es mayor que el trabajar con levaduras comerciales.

Se observa en la Figura 6 las gráficas de superficie que representan los datos obtenidos de humedad, densidad, $\mathrm{pH}$, tiempo y temperatura. Una humedad de $56 \%$, y $\mathrm{pH} 7.6$ favorece el incremento de la temperatura a niveles por encima de los $50^{\circ} \mathrm{C}$. A medida que la humedad $\mathrm{y}$ temperatura disminuyen, el aumento del $\mathrm{pH}$ evidencia reacciones bioquímicas del compostaje en favor de su estabilización p.e. descarboxilación de aniones orgánicos (Cayuela, SánchezMonedero, \& Roig, 2010).
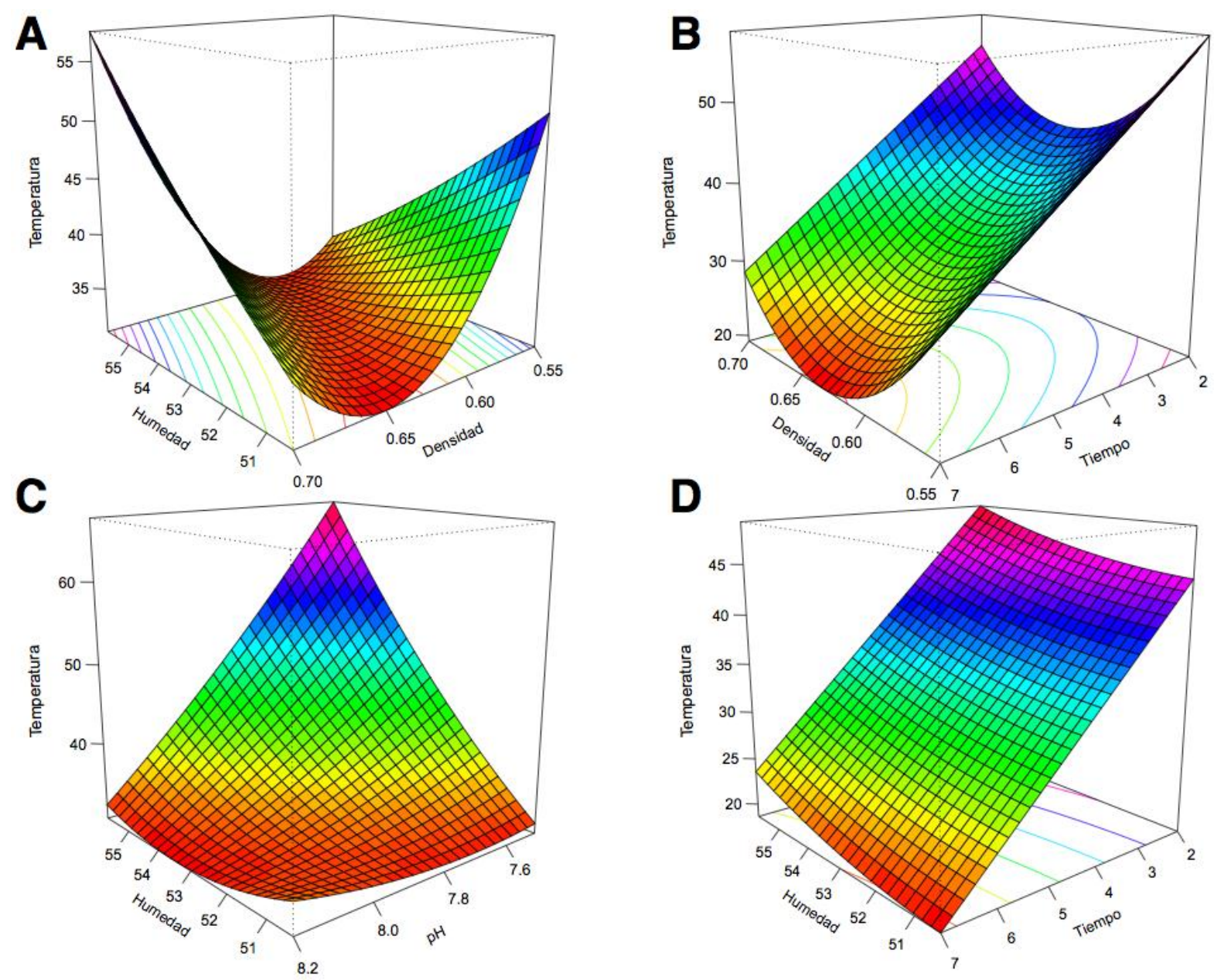

Figura 6. Gráficas de superficie del proceso de compostaje en 7 semanas con un $R^{2}=0.9377$, $\mathrm{p}<0.0001$ a $5 \%$ de significancia. (A) humedad, densidad y temperatura, (B) densidad, tiempo y temperatura, (C) humedad, $\mathrm{pH}$ y temperatura, (D) humedad, tiempo y temperatura.

\section{Conclusiones y Recomendaciones}

En general, todos los cuatro tratamientos en donde se combinan variables de proceso presentan el mismo patrón; sin embargo, se destaca el tratamiento con vegetales y EMAS. Los cambios considerables de temperatura de los cuatro tratamientos de compostaje indican que el proceso de descomposición de los residuos se realizó en un periodo de siete semanas hasta llegar a un 
periodo de madurez. Así, se cumplió con el objetivo de reducir el tiempo de compostaje en comparación con el tiempo tradicional de 16-24 semanas.

Se destaca la formulación de compost obtenida con residuos vegetales y microorganismos aislados de un bosque muy húmedo premontano. En este producto se encontraron concentraciones relevantes de carbono (10.13\%) y nitrógeno (0.96\%). En contenido de materia orgánica, se registró un porcentaje final de $17.48 \%$. Este resultado remarca la importancia de incluir residuos vegetales en el compostaje por su contenido celulósico. El análisis estadístico comprueba la incidencia que origina el tratamiento con EMAS y residuos vegetales en función de la temperatura óptima de compostaje. Tomando en cuenta este tratamiento, se obtuvo un modelo matemático de ajuste polinomial que explica la relación entre el tiempo y temperatura del proceso de compostaje. Este modelo matemático es de importancia para el cálculo de consumo de materia prima y predicción de las condiciones de operación.

\section{Bibliografía}

Adediran, J. A., Taiwo, L. B., \& Sobulo, R. A. (2003). Effect of Organic Wastes and Method of Composting on Compost Maturity, Nutrient Composition of Compost and Yields of Two Vegetable Crops. Journal of Sustainable Agriculture, 22(4), 95-109. doi:10.1300/J064v22n04_08

Agnew, J. M., \& Leonard, J. J. (2003). The Physical Properties of Compost. Compost Science \& Utilization, 11(3), 238-264. doi:10.1080/1065657X.2003.10702132

Antil, R. S., \& Raj, D. (2012). Chemical and microbiological parameters for the characterization of maturity of composts made from farm and agro-industrial wastes. Archives of Agronomy and Soil Science, 58(8), 833-845. doi:10.1080/03650340.2011.554402

Bougnom, B. P., Knapp, B. A., Elhottová, D., Koubová, A., Etoa, F. X., \& Insam, H. (2010). Designer compost with biomass ashes for ameliorating acid tropical soils: Effects on the soil microbiota. Applied Soil Ecology, 45(3), 319-324. doi:10.1016/j.apsoil.2010.05.009

Chantal, K., Shao, X., Jing, B., Yuan, Y., Hou, M., \& Liao, L. (2013). Effects of effective microorganisms (EM) and bio-organic fertilizers on growth parameters and yield quality of flue-cured tobacco (Nicotiana tabacum). Journal of Food, Agriculture \& Environment, 11(2), $1212-1215$.

Insam, H., Franke-Whittle, I., \& Goberna, M. (2009). Microbes at Work: From Wastes to Resources. Springer. Retrieved from http://books.google.com.ec/books?id=5lhHN3Pq2goC 
Insam, H., Franke-Whittle, I., \& Goberna, M. (Eds.). (2010). Microbes at Work. Berlin, Heidelberg: Springer Berlin Heidelberg. Retrieved from http://www.springerlink.com/index/10.1007/9783-642-04043-6

Jusoh, M. L. C., Manaf, L. A., \& Latiff, P. A. (2013). Composting of rice straw with effective microorganisms (EM) and its influence on compost quality. Iranian Journal of Environmental Health Science \& Engineering, 10(1), 17-17.

Organización Panamericana de la Salud. (2002). Análisis sectorial de residuos sólidos. Organización Mundial de la Salud. Retrieved from http://www.bvsde.paho.org/bvsars/e/fulltext/analisis/ecuador.pdf

Restrepo, N. J. F. (2007). Diccionario Ambiental. Ecoe Ediciones. Coleccion Textos Universitarios. Soliz, F. (2011). Retos urgentes para un mundo sin basura. Clinica Ambiental: Alerta Naranja. Retrieved from http://www.clinicambiental.org/docs/publicaciones/Alerta_Desechos.pdf

Termorshuizen, A. J., Moolenaar, S. W., Veeken, A. H. M., \& Blok, W. J. (2004). The value of compost. Reviews in Environmental Science and Bio/Technology, 3(4), 343-347. doi:10.1007/s11157-004-2333-2

Xu, D., Liu, D., Tang, Z., Yu, G., Yuan, J., Shen, Q., \& Huang, Q. (2012). Structure of chemical components in different compost extracts characterized by chromatogram and spectroscopy analysis and its influence on plant growth promotion. Journal of Material Cycles and Waste Management, 14(4), 325-333. doi:10.1007/s10163-012-0071-z 